\title{
ESTUDO RADIOGRÁFICO DO ÍNDICE ACROMIAL E SUA RELAÇÃO COM AS LESÕES DO MANGUITO ROTADOR
}

\author{
RADIOGRAPHIC ANALYSIS OF THE ACROMION INDEX \\ AND ITS ASSOCIATION WITH ROTATOR CUFF TEARS
}

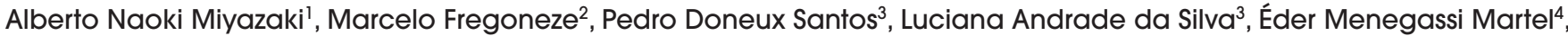
Leandro Gervazoni Debom ${ }^{4}$, Manoel Loyola Andrade ${ }^{4}$, Sérgio Luiz Checchia ${ }^{5}$
\end{abstract}

\section{RESUMO}

Objetivo: O objetivo deste estudo é avaliar a relação da projeção lateral do acrômio com as lesões do manguito rotador (LMR) na população brasileira. Métodos: A projeção lateral do acrômio foi mensurada por meio de radiografias de ombros em sua incidência anteroposterior realizadas com a cavidade glenoidal em seu perfil absoluto e a cabeça do úmero em rotação neutra ou medial. Foi aferido o índice acromial (IA) pela razão entre a distância do plano da cavidade glenoidal ao extremo lateral do acrômio e a distância do plano da cavidade glenoidal ao extremo lateral da cabeça do úmero. Este índice foi mensurado em 83 indivíduos (idade média de 54 anos) com LMR e comparados com um grupo de 28 indivíduos (idade média de 48 anos) que não as apresentavam. A presença ou ausência de LMR foi evidenciada por meio de ressonância magnética. Resultados: O IA médio foi de 0,7194 nos pacientes com LMR e de 0,6677 nos indivíduos que não a apresentavam na população brasileira. Essa diferença foi estatisticamente significativa com um p = 0,001. Conclusão: Pode-se estabelecer relação do IA e lesão do manguito rotador na população brasileira.

Descritores - Bainha rotadora; Acrômio; Radiografia

\section{ABSTRACT}

Objective: The purpose of this study is to evaluate the association between lateral extension of the acromion and rotator cuff tears (RCT) in the Brazilian population. Methods: Lateral extension of the acromion was measured using anteroposterior radiographs of the shoulders, carried out with glenoid cavity in the absolute profile and the humeral head in neutral or internal rotation. The acromion index (AI) was defined by the ratio of the distance from the glenoid cavity to the lateral border of the acromion, and the distance from the glenoid cavity to the lateral border of the humeral head. This index was measured in 83 patients with (average age 54 years) with $R C T$ and compared with a group with 28 patients (average age 48 years) without RCT. The presence or absence of RCT was defined by Magnetic resonance Imaging. Results: The average AI was 0.7194 in the patients with RCT and 0.6677 in the individuals without RCT, in the Brazilian population. This difference was highly significant, with $P<0.001$. Conclusion: An association can be established between AI and rotator cuff tear in the Brazilian population.

Keywords - Rotator cuff; Acrominon; Radiography

\section{INTRODUÇÃO}

A etiologia das lesões do manguito rotador (LMR) ainda é controversa ${ }^{(1)}$; no entanto, elas têm sido relacionadas com o formato do acrômio ${ }^{(2)}$. Bigliani et $a l^{(3)}$, descreveram três tipos de acrômio, relacionando o tipo III (ganchoso) com uma maior prevalência de LMR. Wang e Shapiro ${ }^{(4)}$, bem como Ikemoto et al ${ }^{(5)}$, relataram maior prevalência do acrômio desse tipo em pacientes mais idosos.

Zuckerman et al $^{(6)}$ realizaram estudo morfométrico em ombros de cadáveres, e encontraram maior projeção anterior e menor inclinação do acrômio em cadáveres com LMR comparativamente com os sem lesão. Outro parâmetro de avaliação do formato do acrômio é a angulação lateral deste, estudado por Banas et $a^{(7)}$, em

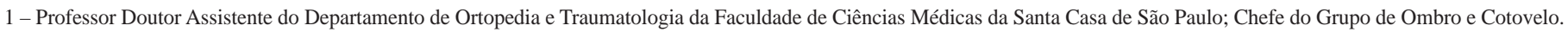

2 - Professor Assistente do Departamento de Ortopedia e Traumatologia da Faculdade de Ciências Médicas da Santa Casa de São Paulo; Assistente do Grupo de Ombro e Cotovelo.

3 - Assistente do Grupo de Ombro e Cotovelo.

4 - Médico Estagiário do Grupo de Ombro e Cotovelo.

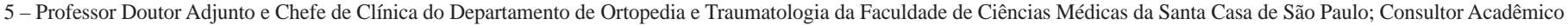
do Grupo de Ombro e Cotovelo.

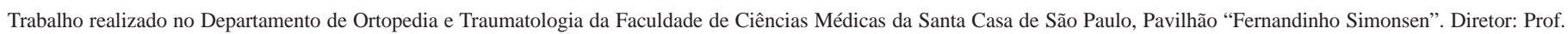
Dr. Osmar Avanzi.

Correspondência: Grupo de Ombro e Cotovelo - Departamento de Ortopedia e Traumatologia - Rua Dr. Cesário Mota Júnior, 112 - Vila Buarque - 01220-020 - São Paulo - SP E-mail: ombro@ombro.med.br 
imagens de ressonância magnética, encontraram ângulos menores em pacientes com LMR.

Analisando o aspecto lateral da forma do acrômio, Nyffeler et $a l^{(2)}$ e Torrens et $a l^{(8)}$ encontraram uma relação direta entre a projeção lateral do acrômio e as LMR. A partir desta relação, Nyffeler et $a l^{(2)}$ propuseram um modelo para justificá-la, no qual o vetor da força muscular resultante do músculo deltoide seria influenciado pela projeção lateral do acrômio. A contração do músculo deltoide durante a abdução ativa tracionaria a cabeça do úmero para cima e também a pressionaria contra a cavidade glenoidal. A orientação do vetor de força resultante depende da orientação das fibras musculares do deltoide na sua origem no acrômio. Quanto mais lateral for a origem no acrômio, maior será o componente ascendente da força resultante, e quanto menor for a projeção lateral do acrômio, maior será o componente compressivo da força contra a cavidade glenoidal (Figura 1). Pode-se imaginar que um maior componente ascendente de força ( $\mathrm{Fa}$ ) favoreça ao impacto subacromial e, como consequência, às alterações degenerativas do tendão do supraespinal, enquanto que uma maior força compressiva (Fc) favoreça às alterações degenerativas na articulação do ombro ${ }^{(2)}$. Porém, esta relação não é consenso na literatura, tendo em vista que Van Nüffel e $\mathrm{Nijs}^{(9)}$, assim como Itoi $^{*}$ não a encontraram em seus estudos, apesar de seus trabalhos terem sido realizados com metodologia semelhante.

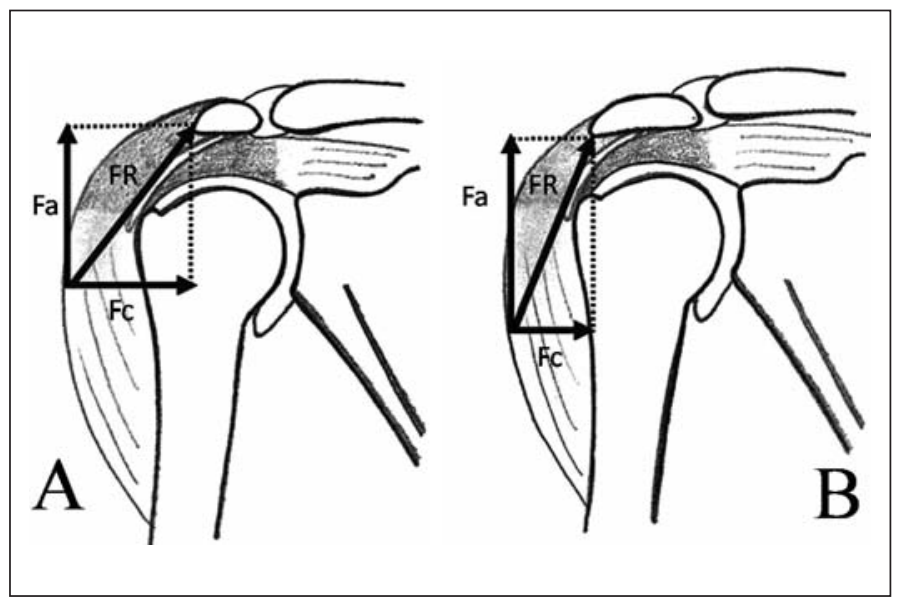

FR - Força resultante; Fa - Força ascendente; Fc - Força compressiva

Figura 1 - A) Representação esquemática da força resultante que atua sobre a cabeça do úmero quando há uma menor extensão lateral do acrômio. B) Representação da força resultante quando a inserção do deltóide é mais lateral

* Comunicação pessoal Professor Itoi Eiji. Búzios, 2008
Nosso trabalho tem como objetivo avaliar, com metodologia também semelhante a Van Nüffel e Nijs ${ }^{(9)} \mathrm{e}$ Itoi ${ }^{*}$, a forma do acrômio, especificamente sua projeção lateral, aferida radiograficamente por meio de um índice, correlacionando este índice com as LMR.

\section{CASUÍSTICA E MÉTODOS}

Foram analisadas radiografias de pacientes operados pelo Grupo de Cirurgia de Ombro e Cotovelo do Departamento de Ortopedia e Traumatologia da Faculdade de Ciências Médicas da Santa Casa de Misericórdia de São Paulo - Pavilhão “Fernandinho Simonsen”, no período de julho de 1995 a dezembro de 2007.

As radiografias do ombro foram padronizadas, sendo utilizadas somente as que haviam sido feitas com correção da anteversão da cavidade glenoidal; o membro superior foi radiografado em posição de repouso junto ao corpo, com a região proximal do úmero em rotação neutra ou medial. Segundo estudo publicado por Nyffeler et $a l^{(2)}$, não há diferença em mensurar o índice acromial com o ombro em rotação neutra ou medial.

Nessas imagens radiográficas foram feitas duas aferições, tomando-se como referência o plano da cavidade glenoidal, a extremidade lateral da cabeça do úmero e a extremidade lateral do acrômio. A distância entre a extremidade lateral do acrômio e o plano da cavidade glenoidal foi chamada de GA. A distância entre a extremidade lateral do úmero e o plano da cavidade glenoidal foi chamada de GU. A razão entre os valores de GA e GU determina um índice, denominado índice acromial (IA) (Figura 2).

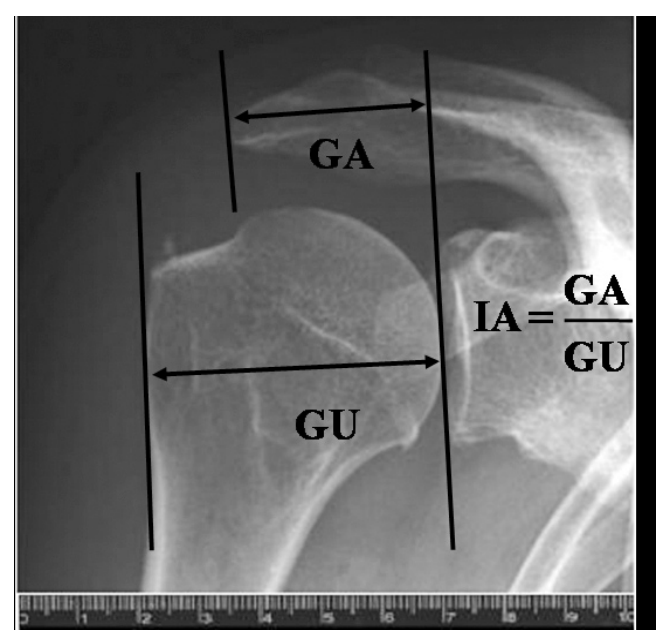

Figura 2 - Imagem radiográfica de ombro direito, na incidência anteroposterior, após ser escaneada com régua milimetrada utilizada para calibrar o programa. Nela está demonstrado o índice acromial (IA), obtido pela razão entre as distâncias cavidade glenoidal e borda lateral do acrômio (GA) e cavidade glenoidal e borda lateral do úmero (GU) 
Não houve preocupação quanto à distância entre a ampola do aparelho de raio-X e o filme da radiografia, pois se trata de um índice e as alterações dos parâmetros não interferem no resultado. Para certificar-se que a variação na inclinação da ampola de raio-X não alteraria as medidas do IA, criamos um grupo controle de 10 pacientes; que foram submetidos a radiografias centradas na cavidade glenoidal em anteroposterior com angulações de $0^{\circ}, 30^{\circ}$ de inclinação caudal e $30^{\circ}$ de inclinação cranial. Foi medido o IA nessas incidências e submetidas à análise estatística (teste de Friedman). A análise deste índice, nas três incidências radiográficas, mostrou valores semelhantes e um p-valor de 0,999, concluindo que não houve influência da inclinação da ampola de raio-X no resultado (Tabela 1 ).

Como critérios de inclusão foram selecionados pacientes que possuíam lesão completa do manguito rotador, comprovadas por imagens de ressonância magnética e durante a cirurgia. Como grupo controle, foram incluídos pacientes tratados por doença no ombro, e que não possuíam LMR, em imagens de ressonância magnética. Definimos como critério de exclusão e de não inclusão aqueles que tivessem antecedentes de fraturas da cintura escapular, artrite ou artrose degenerativa, osteonecrose e sequela de infecção.

Os pacientes selecionados foram divididos em dois grupos: grupo I - que possuía lesão completa do manguito rotador (83 casos); e grupo II - com o manguito rotador íntegro (28 casos) (Tabela 2).

As radiografias foram digitalizadas em scanner HP modelo Deskjet F4180 ${ }^{\circledR}$. Estas imagens foram analisadas com o uso do programa Image J 1.41 (Wayne Rasband, Research Services Branch, National Institute of Mental Health, Bethesda, Maryland, USA) disponível para download no site http://rsbweb.nih.gov; que permite uma medição precisa de distâncias em figuras, a partir de um parâmetro para calibragem. Foi utilizada uma régua milimetrada como parâmetro para calibração (Figura 2).

Os dados obtidos foram submetidos à análise estatística por meio do teste $t$ de Student, controlado pelo teste de Levene para igualdade de variâncias, com nível de
Tabela 2 - Distribuição dos grupos I e II em relação ao sexo, média de idade e lado acometido

\begin{tabular}{c|c|c}
\hline & Grupo I & Grupo II \\
\hline $\mathrm{n}$ & 83 & 28 \\
\hline Média de idade (anos) & $54(32-77)$ & $48(35-63)$ \\
\hline & 30 homens & 12 homens \\
Sexo & $(36 \%)$ & $(43 \%)$ \\
& 53 mulheres & 16 mulheres \\
& $(64 \%)$ & $(57 \%)$ \\
\hline & 65 direitos & 18 direitos \\
Lado & $(78 \%)$ & $(64 \%)$ \\
& 18 esquerdos & 10 esquerdos \\
& $(22 \%)$ & $(36 \%)$ \\
\hline Fonte: SAME-DOT ISCMSP & \multicolumn{2}{c}{} \\
$\mathrm{n}=$ número total de pacientes &
\end{tabular}

$\mathrm{n}=$ número total de pacientes

significância de 5\%. Também foi utilizado o teste do Quiquadrado, no intuito de verificarmos uma possível diferença na distribuição dos sexos nos grupos estudados.

\section{RESULTADOS}

Nos pacientes do grupo I encontrou-se uma média de idade de 54 anos, variando de 32 a 77 anos, enquanto que no grupo II essa média foi de 48 anos, variando de 35 a 63 anos. No grupo I houve um predomínio de indivíduos do sexo feminino, correspondendo a 64\% (53 mulheres), fato que também ocorreu no grupo II, no qual 57\% dos pacientes eram do sexo feminino (16 mulheres). Entretanto essa diferença na distribuição dos sexos nos grupos I e II não foi estatisticamente significante $(p=0,527)$ (Tabela 2).

Quanto ao lado, houve um predomínio do lado direito em ambos os grupos, com $78 \%$ dos ombros no grupo I e $64 \%$ dos ombros no grupo II (Tabela 2).

Verificamos um IA médio de 0,7194 nos indivíduos que apresentavam LMR (grupo I); e nos indivíduos que possuíam manguito rotador íntegro (grupo II) de 0,6677, cuja análise estatística mostrou um $\mathrm{p}=0,001$, ou seja, há uma relação estatisticamente significante entre lesão de manguito rotador e uma maior projeção lateral do acrômio.

Tabela 1 - Variação do índice acromial conforme incidência radiográfica

\begin{tabular}{|c|c|c|c|c|c|c|c|c|c|}
\hline Índice acromial & $\mathrm{n}$ & Média & Desvio padrão & Mínimo & Máximo & Mediana & Percentil 25 & Percentil 75 & $P$ valor \\
\hline $30^{\circ}$ cranial & 10 & 0,66309 & 0,09457 & 0,51820 & 0,80240 & 0,66585 & 0,59290 & 0,73233 & \\
\hline $0^{\circ}$ & 10 & 0,66489 & 0,09368 & 0,50930 & 0,79950 & 0,67545 & 0,59438 & 0,73433 & $>0,999$ \\
\hline $30^{\circ}$ caudal & 10 & 0,66434 & 0,09338 & 0,52780 & 0,80230 & 0,65980 & 0,59245 & 0,74283 & \\
\hline
\end{tabular}




\section{DISCUSSÃO}

Em 1972, foi Neer ${ }^{(10)}$ quem melhor estudou a síndrome do impacto, identificando que o impacto da porção inferior do acrômio, juntamente com o ligamento coracoacromial e a superfície inferior do ligamento acromioclavicular, eram os responsáveis pelo estreitamento do espaço subacromial, o que levava à lesão dos tendões. Entretanto, hoje, sabemos que a patogênese das LMR é provavelmente multifatorial ${ }^{(1)}$. Uma das possíveis causas desta lesão é uma maior projeção lateral do acrômio, como proposto por Nyffeller $e t a l^{(2)}$. Porém, esta associação não é consenso na literatura, como é a projeção anterior do acrômio ${ }^{(3,6,7,10)}$.

Nossos resultados apoiam a teoria de Nyffeler et $a l^{(2)}$, pois, em indivíduos portadores de LMR, encontramos um IA maior, ou seja, uma maior projeção lateral do acrômio. Essa forma do acrômio faz com que o deltoide tenha origem mais lateral, originando uma força resultante com orientação mais ascendente (Fa), favorecendo provavelmente o impacto subacromial. Nos pacientes com o manguito rotador íntegro esse índice foi menor, ou seja, uma menor projeção lateral do acrômio, com uma força resultante mais orientada no sentido de fazer uma compressão (Fc) contra a cavidade glenoidal. Embora não tivesse sido o objetivo de nosso estudo, não encontramos sinais de artrose na articulação do ombro nesses pacientes como supõe esta teoria. Nossos achados, de certa forma também corroboram os resultados de Torrens et $a l^{(8)}$, pois, apesar da projeção lateral do acrômio ser calculada de maneira diferente, ou seja, por um outro índice, também encontramos a relação entre uma maior projeção lateral do acrômio com LMR.

Por outro lado, nossos resultados e os de Nyffeler et $a l^{(2)}$ não são os mesmos de Van Nüffel e $\mathrm{Nijs}^{(9)}$ e de Itoi $^{*}$, apesar de utilizarmos a mesma maneira de mensurar a projeção lateral do acrômio e de os grupos serem semelhantes quanto ao sexo e idade. A única diferença presente é o fato de tanto o grupo com LMR quanto o grupo controle de Itoi ${ }^{*}$ serem formados na sua maioria por indivíduos do sexo masculino, o que poderia ser um viés devido ao fato de existirem relatos de que as LMR

\section{REFERÊNCIAS}

1. Uhtof HK, Loehr J, Sarkar K. The pathogenesis of rotator cuff tears. The shoulder. In: Proceedings of the Third International Conference on Surgery of the Shoulder, Fukuoka - Japan; 1986. p. 211-2.

2. Nyffeler RW, Werner CM, Sukthankar A, Schmid MR, Gerber C. Association of a large lateral extension of the acromion with rotator cuff tears. J Bone Joint Surg Am. 2006;88(4):800-5.

3. Bigliani LU, Morrison DS, April EW. The morphology of the acromion and its relationship to rotator cuff tears. Orthop Trans. 1986;10:216.

4. Wang JC, Shapiro MS. Changes in acromial morphology with age. J Shoulder Elbow Surg. 1997;6(1):55-9.

5. Ikemoto RY, Bezerra AD, Monte FA, Telles RB, Fujiki EN, Porto LCK. Acrômio em forma de gancho: uma variação anatômica ou um processo degenerativo? Rev Bras Ortop. 2005;40(8):454-63.

6. Zuckerman JD, Kummer FJ, Cuomo F, Simon J, Rosenblum S, Katz N. The in- seriam mais prevalentes no sexo feminino, porém isso também não é consenso na literatura ${ }^{(11)}$.

Os indivíduos selecionados com LMR e com o manguito rotador íntegro, tanto em estudos anteriores quanto no nosso, têm uma média de idade entre 45 e 65 anos, ou seja, estariam na fase III da síndrome do impacto, descrita por Neer ${ }^{(12)}$, quando ocorrem as rupturas totais do manguito rotador.

Outro fator que poderia influenciar os resultados, seria uma não padronização das imagens radiográficas utilizadas para a mensuração do IA. Porém, tivemos o cuidado de selecionar radiografias com correção da anteversão da cavidade glenoidal; e em que o úmero estivesse em rotação neutra ou medial, como Nyffeler et $a^{(2)}$ já demonstraram previamente, pois assim não haveria influência no IA em ambas posições. Torrens et al ${ }^{(8)}$ já relataram que a distância da ampola ao filme, no momento da realização da radiografia, também não altera o IA, pois este é determinado pela razão entre duas medidas mensuradas na mesma imagem radiográfica. Em nosso estudo demonstramos que a angulação da ampola do raio-X também não influencia na determinação do IA, evitando esse viés na realização de nosso estudo.

Um fator que deve ser levado em consideração é que Itoi $^{*}$ realizou a mensuração na população japonesa, Nyffeler et $a l^{(2)}$ na população suíça, Van Nüfell e Nijs ${ }^{(9)}$ na população belga e nosso estudo foi realizado na população brasileira. Como cada raça possui características próprias, seria essa diferença entre os resultados determinada por um fator relacionado ao biótipo de cada raça? Porém, para investigar melhor essa questão, outros estudos devem ser realizados, levando-se em consideração outros parâmetros morfológicos, como o tipo do acrômio, ângulo lateral e a inclinação anterior do acrômio, na tentativa de se encontrar o fator etiológico preciso das LMR.

\section{CONCLUSÃO}

Concluímos que as LMR podem estar associadas a um IA maior, ou seja, a uma maior projeção lateral do acrômio. fluence of coracoacromial arc anatomy on rotator cuff tears. J Shoulder Elbow Surg. 1992;1(1):4-14.

7. Banas MP, Miller RJ, Totterman S. Relationship between the lateral acromion angle and rotator cuff disease. J Shoulder Elbow Surg. 1995;4(6):454-61.

8. Torrens C, López JM, Puente I, Cáceres E. The influence of the acromial coverage index in rotator cuff tears. J Shoulder Elbow Surg 2007;16(3):347-51.

9. Van Nuffel M, Nijs S. Acromial morphology, acromial index and rotator cuff problems: is there a correlation? The Pellenberg Orthopaedic Yearbook. 2006-2007:37-44.

10. Neer CS 2 nd. Anterior acromioplasty for the chronic impingement syndrome in the shoulder: a preliminary report. J Bone Joint Surg Am. 1972;54(1):41-50.

11. Jerosch J, Müller T, Castro WH. The incidence of rotator cuff rupture. An anatomic study. Acta Orthop Belg. 1991;57(2):124-9.

12. Neer CS 2nd. Impingement lesions. Clin Orthop Relat Res. 1983;(173):71-7. 